\title{
Group-Wise Diffeomorphic Diffusion Tensor Image Registration
}

\author{
Xiujuan Geng, Hong Gu, Wanyong Shin, \\ Thomas J. Ross, and Yihong Yang \\ National Institute on Drug Abuse, NIH \\ gengx@nida.nih.gov
}

\begin{abstract}
We propose an unbiased group-wise diffeomorphic registration technique to normalize a group of diffusion tensor (DT) images. Our method uses an implicit reference group-wise registration framework to avoid bias caused by reference selection. Log-Euclidean metrics on diffusion tensors are used for the tensor interpolation and computation of the similarity cost functions. The overall energy function is constructed by a diffeomorphic demons approach. The tensor reorientation is performed and implicitly optimized during the registration procedure. The performance of the proposed method is compared with reference-based diffusion tensor imaging (DTI) registration methods. The registered DTI images have smaller shape differences in terms of reduced variance of the fractional anisotropy maps and more consistent tensor orientations. We demonstrate that fiber tract atlas construction can benefit from the group-wise registration by producing fiber bundles with higher overlaps.
\end{abstract}

\section{Introduction}

Diffusion tensor magnetic resonance imaging (DTI) is an emerging imaging modality to non-invasively measure water diffusion in biological tissues [1]. It plays an important role in studying brain white matter microstructure and anatomical connectivity. DTI registration is necessary to estimate correspondences among different diffusion tensor (DT) images, study white matter alterations in developing and disease populations, and build a white matter atlas. DTI registration techniques have been first proposed in 2. In contrast to scalar images, DT images are multidimensional at each voxel, and more information such as shape and orientation of the tensors can be used in registration. Recently, many DTI registration methods have been presented using whole tensors 34 , features extracted from tensors [56, multi-channels [7], and multi-contrasts [8].

In building white matter and fiber tract atlases, group-wise registration is crucial to map each image to an unbiased common space [9]. In [10, the fractional anisotropy (FA) extracted from DTI is used and registered to a common space following the work in [11. Zhang et al. 12] proposed an atlas construction method using the information encoded in tensors, especially the orientation information, which may enable more accurate alignment of fiber tracts. This method estimates the unbiased atlas iteratively. The input DT images are first 
averaged, and each image is registered to it. All deformed images are averaged again to get the second atlas; the procedure is repeated until the atlas converges [13. Barmpoutis et al. 14 recently developed a group-wise registration of 4th-order tensor images and showed the improvement compared to 2nd-order tensor registration using synthetic fiber crossing data.

In this paper, we propose a group-wise DTI registration technique that extends the implicit-reference group-wise (IRG) registration framework proposed by Geng et al. [15]. The DTI IRG registration simultaneously estimates diffeomorphic transformations and the deformed DT images converge to the group average. The unbiased atlas can be obtained by averaging the deformed images. Tensor reorientation is applied using "Finite Strain" strategy [2] during the estimation to preserve the geometric features of the tensor fields [9]. The Log-Euclidean metric is used to define the similarity cost and for tensor interpolation. Compared to the work in [12, our method does not compute the average input images as the reference and update the atlas by repeating the registration multiple times. We jointly estimate transformations that deform each pair of DT images to have similar shapes. Another major difference is that the registration algorithm in [12] estimates transformations using an enhanced piecewise affine framework, our registration adapts large deformation diffeomorphic demons approach [4.

The FA map variance and the directional consistency after the IRG DTI registration were compared to reference-based registration. Results show smaller registration errors using the group-wise registration. To demonstrate the advantage in building a fiber tract atlas, we constructed the uncinate fasciculus (UF) atlas of a group of healthy normal subjects using group-wise and reference DTI registration methods. The proposed group-wise registration produces higher bundle overlap and therefore a sharper UF atlas.

\section{Implicit-Reference Group-Wise DTI Registration}

Tensor distance computation, interpolation and reorientation are involved in DTI registration. The Affine-invariant metric, a Riemannian metric, is the natural metric for diffusion tensors that are positive semi-definite. Here we use Log-Euclidean metric since it is computationally efficient and provides similar performance compared to Riemannian metric [16]. The distance between two tensors is defined as:

$$
D\left(T_{i}, T_{j}\right)=\left(\operatorname{Trace}\left(\log T_{i}-\log T_{j}\right)^{2}\right)^{\frac{1}{2}} .
$$

The $\log$ of a rotated tensor can be calculated as $\log \left(R^{T} T R\right)=R^{T} \log (T) R$. The interpolation of log tensors can be performed using the Euclidean metric after taking the log transformation of the tensors. These properties make the registration convenient by applying a log transform of the input DTI data before registration, using the Euclidean metric to estimate the transformations and performing exponential transform to convert log tensors back to tensors. 


\subsection{Algorithm Overview}

The scalar-based IRG registration with a small deformation elastic model can be stated as an optimization problem of finding a set of $N$ transformations that deform $N$ images to an implicit reference by minimizing the following function:

$$
C=\sum_{i=, 1}^{N} \sum_{j=1}^{i-1} C_{S i m}\left(I_{i}\left(\Phi_{i S}\right), I_{j}\left(\Phi_{j S}\right)\right)+\sum_{i=1}^{N} C_{R e g}\left(\Phi_{i}\right) .
$$

$\Phi_{i S}$ is the transformation to be estimated to map image $I_{i}$ to the implicit reference $I_{S} . C_{S i m}$ is the similarity cost between deformed $I_{i}$ and $I_{j} . C_{R e g}$ is the regularization cost and can be defined as an elastic differential operator [15].

To map DT images with large shape differences, a similar framework as diffeomorphic demons was used to estimate transformations in diffeomorphisms. Unlike the work in [4, the exact finite strain differential is not implemented here, and the diffeomorphic transformations are ensured by constraining velocity fields small enough at each step. The optimization scheme is to separate the energy function to two parts by introducing another transformation variable $\Psi$. The minimization of the similarity and regularization costs can be separated. A quadratic regularization can be performed efficiently with a convolution kernel.

The group-wise diffeomorphic DTI registration is formulated as estimating a set of transformations that minimize the cost function:

$$
C=\sum_{i=1}^{N} \sum_{j=1}^{i-1} C_{S i m}\left(T_{i} \circ \Phi_{i S}^{t}, T_{j} \circ \Phi_{j S}^{t}\right)+\sum_{i=1}^{N} D\left(\Phi_{i S}^{t}, \Psi_{i S}^{t}\right)^{2}+\sum_{i=1}^{N} C_{R e g}\left(\Psi_{i S}^{t}, v_{i S}^{t}\right),
$$

where $v_{i S}^{t}$ is the velocity field from $T_{i}$ to the implicit reference at time $t, \Phi_{i S}^{t}=$ $\int_{\tau=0}^{t} v_{i S}^{\tau} d \tau, D\left(\Phi_{i S}^{t}, \Psi_{i S}^{t}\right)$ is the distance between $\Phi$ and $\Psi$ to constrain $\Psi$ close to $\Phi$, and the last term is the regularization term to ensure smooth velocity and transformation fields, which is defined as an isotropic differentiable operator $\nabla(\cdot)$ in this work. The similarity cost can be defined as the squared Euclidean distance after $\log$ transform of the tensors. Let $T_{i}$ denote the log transformed tensor image for simplicity. The cost function in Eq.(2) can be rewritten as:

$$
\begin{aligned}
& \sigma \sum_{i=1}^{N} \sum_{j=1}^{i-1} \int_{\Omega}\left\|T_{i} \circ \Phi_{i S}^{t}(x)-T_{j} \circ \Phi_{j S}^{t}(x)\right\|^{2} d x+\rho \sum_{i=1}^{N} \int_{\Omega}\left\|\Phi_{i S}^{t}(x)-\Psi_{i S}^{t}(x)\right\|^{2} d x \\
& +\sum_{i=1}^{N}\left(\lambda_{\Psi} \int_{\Omega}\left\|\nabla\left(\Psi_{i S}^{t}(x)\right)\right\|^{2} d x+\lambda_{v} \int_{\Omega}\left\|\nabla\left(v_{i S}^{t}(x)\right)\right\|^{2} d x\right)
\end{aligned}
$$

where $\Omega$ represents the tensor image space, and $\sigma, \rho, \lambda_{\Psi}$ and $\lambda_{v}$ are weighting parameters for each cost term. We set $\sigma$ to $\frac{1}{N-1} \sum_{j=1, \neq i}^{N} \frac{1}{\left\|T_{i} \circ \Phi_{i S}^{t}(x)-T_{j} \circ \Phi_{j S}^{t}(x)\right\|^{2}}$ for each $\Phi_{i S}$, varied $\rho$ to keep the largest update field less than 1 at each iteration, and set $\lambda_{\Psi}$ and $\lambda_{v}$ to be 0.5 and 0.5 .

The deformed tensor image $T_{i} \circ \Phi_{i S}^{t}$ can be computed as:

$$
R_{i S} T_{i}\left(\Phi_{i S}^{t}\right) R_{i S}^{T}
$$


where $R_{i S}$ is the reorientation matrix extracted from the Jacobian of the inverse $\Phi_{i S}$ in Eulerian space, or from the Jacobian and taking its transpose afterwards [2]:

$$
R=\left(\left(J(\Phi) \cdot J(\Phi)^{T}\right)^{-\frac{1}{2}} J(\Phi)\right)^{T} .
$$

\subsection{Optimization of the Cost Function}

After linear approximation of the similarity cost function, the first two terms in Eq.(3) become

$$
\begin{aligned}
& \sigma\left\|\sum_{j=1, \neq i}^{N}\left(T_{i} \circ \Psi_{i S}^{t}-T_{j} \circ \Psi_{j S}^{t}\right)+\nabla T_{i} \circ \Psi_{i S}^{t} \cdot v_{i S}^{t}\right\|^{2}+\rho \int_{\Omega}\left\|\Phi_{i S}^{t}(x)-\Psi_{i S}^{t}(x)\right\|^{2} d x \\
& =\left\|\left[\begin{array}{c}
\sum_{j=1, \neq i}^{N} \sqrt{\sigma}\left(T_{i} \circ \Psi_{i S}^{t}-T_{j} \circ \Psi_{j S}^{t}\right) \\
0
\end{array}\right]+\left[\begin{array}{c}
\sqrt{\sigma} \nabla T_{i} \circ \Psi_{i S}^{t} \\
\sqrt{\rho} I
\end{array}\right] v_{i S}^{t}\right\|^{2}
\end{aligned}
$$

where $I$ denotes the identity transformation field. The update of $v_{i S}^{t}$ can be calculated by setting the above equation to zero and solving for $v_{i S}^{t}$ :

$$
v_{i S}^{t}=\frac{-\sum_{j=1, \neq i}^{N}\left(\nabla T_{i} \circ \Psi_{i S}^{t}\right)^{\prime}\left(T_{i} \circ \Psi_{i S}^{t}-T_{j} \circ \Psi_{j S}^{t}\right)}{\left\|\nabla T_{i} \circ \Psi_{i S}^{t}\right\|^{2}+\frac{\rho}{\sigma} I} .
$$

The optimization of the Eq.(3) except the similarity term can be done by convolving a Gaussian kernel with $v_{i S}$ and $\Psi_{i S}$ alternatively [17. Smoothing $v_{i S}$ is more fluid like registration, and smoothing $\Psi_{i S}$ is more diffusion like registration. The iterative process is done for each image simultaneously. The cost function is similar to a recent $4 \mathrm{D}$ registration study with multichannel diffeomorphic Demons algorithm[18].

The implementation of the algorithm can be summarized as follows: (1) take the $\log$ transform of the input tensor images; (2) initialize $\Phi_{i S}$ and $\Psi_{i S}$ to be identity fields; (3) reorient $T_{i}$ using Eqs.(4) (5); (4) let $\Phi_{i S}^{n}=\Psi_{i S} \circ\left(I+v_{i S}^{n}\right)$ and estimate the velocity field $v_{i S}^{n+1}$ according to Eq.(7); (5) regularize $v$ by taking the Gaussian kernel of it: $v_{i S}^{n+1}=\lambda_{v} K * v_{i S}^{n+1} ;(6)$ let $\Psi_{i S}^{n+1}=\lambda_{\Psi} K * \Psi^{n} \circ\left(I+v_{i S}^{n+1}\right)$, and $\Phi_{i S}^{n+1}=\Psi_{i S}^{n+1} ;(7)$ repeat steps 3-6 until convergence, $C_{\text {Sim }}\langle\epsilon$, or $n\rangle$ $N_{\max } ;(8)$ apply the exponential transform to convert the log tensors back to tensors and apply the estimated $\Phi_{i S}$ to them to generate deformed DT images.

\section{Experiments and Results}

We demonstrate the performance of the proposed method using 10 DT images acquired from a 3T Siemens MRI scanner. An EPI-based spin echo sequence was used to acquire diffusion-weighted images (DWIs). The whole brain was covered with 35 axial slices and a $128 \times 128$ in-plane matrix, resulting in a resolution of $1.72 \times 1.72 \times 4.0 \mathrm{~mm}^{3}$. Besides one non-diffusion weighted reference image, 12 directions were used to apply the diffusion-sensitive gradients with a b-factor of 
$1000 \mathrm{~s} / \mathrm{mm}^{2}$. For EPI, TR/TE $=5000 / 87 \mathrm{~ms}, \mathrm{BW}=1700 \mathrm{~Hz} / \mathrm{Pixel}$, and NEX=4. The DT images were reconstructed from DWIs. B0 maps were used to affine align each map to the average of the original maps, and the transformation matrices were applied to the DT images to obtain affine aligned images.

To compare the IRG DTI registration with reference-based methods, each DT image was selected as the reference, and all other images were registered to it using the same diffeomorphic DTI registration framework with the same parameter settings. Therefore, 10 groups of reference-based registrations were performed and compared with the IRG registration. The standard deviation (STD) of FA maps and the directional consistency (DC) of tensor images after registration were computed to evaluate different methods. The FA and DC were computed from the deformed tensor images. The DC is defined as the absolute value of the dot product of the major eigenvectors of two tensor images, ranging from 0 (two directions are perpendicular) to 1 (directions are consistent).

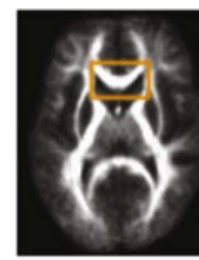

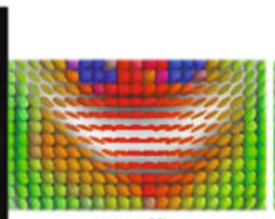

(a) Affine

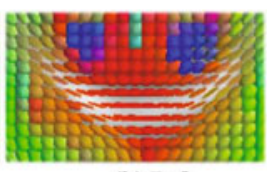

(b) Ref

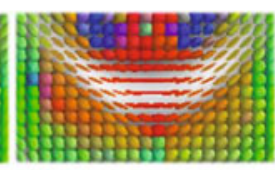

(c) IRG

Fig. 1. Average tensor images in the genu of the corpus callosum after (a)affine alignment, (b) reference-based, and (c) group-wise DTI registration

Fig 1 shows a subregion of the averaged tensor images in the genu of the corpus callosum before diffeomorphic registration (after affine), after a typical referencebased and the proposed IRG DTI registration. The average (using Log-Euclidean metric) of the deformed tensor images has pointier shapes after diffeomorphic registration which may be due to the more consistent directions of the deformed tensors. Compared to the reference-based method, the IRG method produced an average shape closer to the average of the affine aligned images.

Fig 2 plots the STD of FA maps and the DC maps after affine, a typical reference-base and IRG DTI registration. The maps are shown on a mask generated from thresholding the average of all deformed FA maps after all registrations at 0.3. The same mask was used to compute the RMS of the STD of FA maps and the average of DC maps for 10 reference-based registrations and the groupwise registration. The RMS of each registration method is listed in Tab 1. The IRG method produced the least FA variance compared to all reference-based methods. For each registration, nine DC maps were generated and the average of each DC map is plotted in Fig 3 . To compare the registration with image $I_{i}$ as the reference, the DC map was computed between the deformed $I_{i}$, and all other deformed images after IRG registration. The IRG registered tensors with higher directional consistency compared to all reference-based registrations with inconsistent performances varying according to the reference selection. 


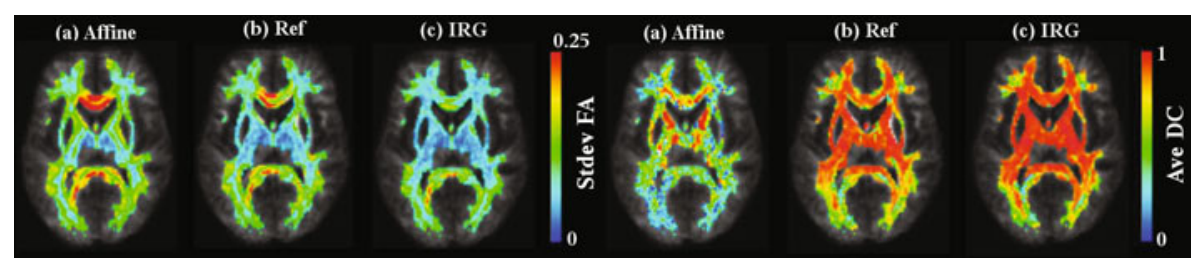

Fig. 2. Standard deviation of FA and DC maps after (a) affine alignment, (b) referencebased, and (c) group-wise DTI registration

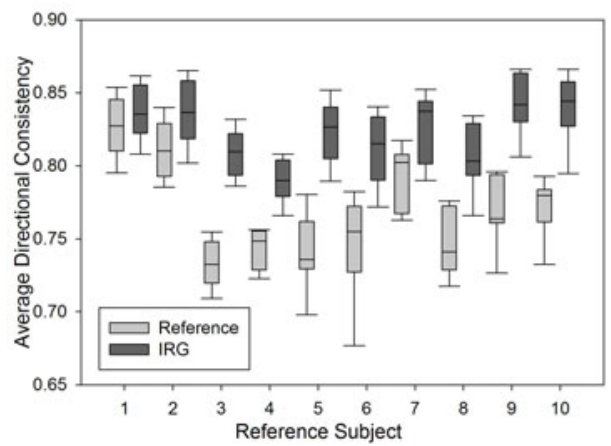

Fig. 3. Average directional consistency values after 10 reference-based registrations and the group-wise registration

The construction of an UF atlas was used to demonstrate the advantage of the group-wise DTI registration. Whole brain white matter fiber tracts were generated using the software "Trackvis" [19] from the registered DTI data. An anatomical atlas provided in AFNI 20 was used to provide the ROIs of right amygdala and medial prefrontal cortex ( $\mathrm{mPFC})$. The anatomical atlas was affine aligned to the average of the deformed FA maps. The ROIs were diluted $2 \mathrm{~mm}$ along the boundary to overlap with the white matter tracts. The UF bundles were extracted for each subject by selecting fibers passing both right amygdala and $\mathrm{mPFC}$. All points on the fiber tracks were projected to the image grid. The number of points in each voxel was counted and the values in each voxel were normalized to 0 to 1 , where 0 means no fiber passing the voxel and 1 indicates this voxel has the highest fiber density. We defined the fiber bundle to be the regions

Table 1. RMS of the standard deviation of the deformed FA maps after reference and group-wise registration

\begin{tabular}{|c|c|c|c|c|c|c|c|c|c|c|c|}
\hline method & ref-1 & ref-2 & ref-3 & ref-4 & ref-5 & ref-6 & ref-7 & ref-8 & ref-9 & ref-10 & IRG \\
\hline RMS & .0933 & .0955 & .111 & .108 & .114 & .115 & .103 & .108 & .111 & .108 & .0889 \\
\hline
\end{tabular}



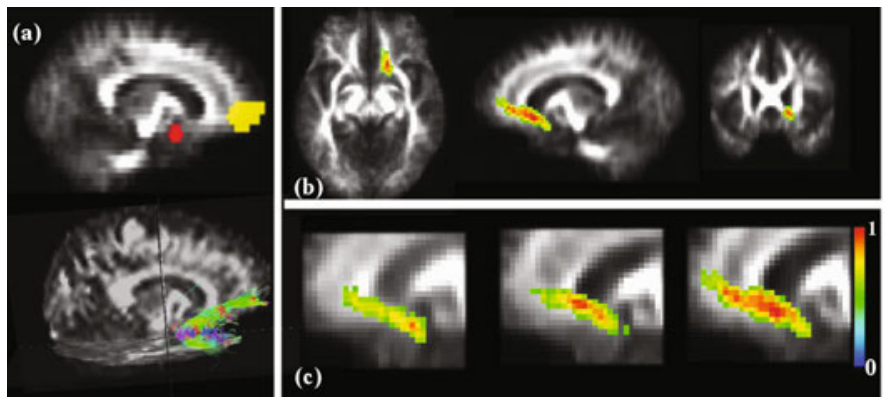

Fig. 4. Uncinate fasciculus atlas generated using group-wise and reference-based DTI methods. (a) Amygdala and $\mathrm{mPFC}$ and the UF fibers from a single subject. (b) The UF atlas built from group-wise registration. (c) The sagittal view of the UF atlas built by (from left to right): affine alignment, reference-based registration, and group-wise registration. The color represent the relative overlap of fiber bundles.

with the fiber density greater than 0.5 . The fiber bundle atlas was computed by averaging each binarized fiber bundle. To evaluate the fiber bundle atlas, the relative overlap at each voxel $x$ was computed: $R O(x)=n(x) / N$, where $\mathrm{n}(\mathrm{x})$ is the number of subjects that have fibers at $\mathrm{x}$, and $\mathrm{N}$ is the total number of subjects. Fig 4 shows the UF atlas after affine, a typical reference-based and IRG registration. The average overlaps of the UF bundles were $0.531,0.616$ and 0.632 after affine, reference-based and IRG methods, respectively.

\section{Discussion}

Overall, our results show that the IRG DTI registration provides better performance in terms of smaller FA variation and higher tensor directional consistency compared to reference-based methods under the same diffeomorphic registration framework. Using the UF as an example, the group-wise registration provides higher overlap of the deformed UF bundles, and therefore improves the construction of the fiber tract atlas.

Even though the unbiased group-wise DTI registration produces less error than the pair-wise reference-based method, it is still difficult to match fiber tracts across subjects due to the large variations and sometimes different topologies of the same structure. Combining fiber bundle information in the registration procedure may help to produce better fiber bundle alignment [21]. Since 2nd order tensors have limitations in representing intra-voxel fiber crossings, tensorbased registrations may not map the complex white matter structures accurately. Higher order tensor registration [14] and registration of orientation distribution functions 22] have the potential to overcome these difficulties.

Acknowledgment. This work was sponsored by the National Institute on Drug Abuse, Intramural Research Program, National Institutes of Health. 


\section{References}

1. Basser, P.J., Pierpaoli, C.: Microstructural and physiological features of tissues elucidated by quantitative-diffusion-tensor mri. Journal of Magnetic Resonance Series B,111, 209-219 (1996)

2. Alexander, D., Pierpaoli, C., Basser, P., Gee, J.: Spatial transformations of diffusion tensor magnetic resonance images. IEEE Trans. Med. Imaging 20(11), 1131-1139 (2001)

3. Chiang, M.C., Leow, A.D., Klunder, A.D., Dutton, R.A., Barysheva, M., Rose, S.E., McMahon, K.L., de Zubicaray, G.I., Toga, A.W., Thompson, P.M.: Fluid registration of diffusion tensor images using information theory. IEEE Trans. Med. Imaging 27(4), 442-456 (2008)

4. Yeo, B.T., Vercauteren, T., Fillard, P., Peyrat, J.M., Pennec, X., Golland, P., Ayache, N., Clatz, O.: Dt-refind: Diffusion tensor registration with exact finitestrain differential. IEEE Trans. Med. Imaging 99 (2009)

5. Yap, P.T., Wu, G., Zhu, H., Lin, W., Shen, D.: Timer: Tensor image morphing for elastic registration. NeuroImage 47, 549-563 (2009)

6. Li, H., Xue, Z., Guo, L., Wong, S.T.: Simultaneous consideration of spatial deformation and tensor orientation in diffusion tensor image registration using local fast marching patterns. In: Prince, J.L., Pham, D.L., Myers, K.J. (eds.) Information Processing in Medical Imaging. LNCS, vol. 5636, pp. 63-75. Springer, Heidelberg (2009)

7. Park, H.J., Kubicki, M., Shenton, M.E., Guimond, A., McCarley, R.W., Maierd, S.E., Kikinis, R., Jolesz, F.A., Westin, C.F.: Spatial normalization of diffusion tensor mri using multiple channels. NeuroImage 20(4), 1995-2009 (2003)

8. Ceritoglu, C., Oishi, K., Li, X., Chou, M.C., Younes, L., Albert, M., Lyketsos, C., van Zijl, P.C., Miller, M.I., Mori, S.: Multi-contrast large deformation diffeomorphic metric mapping for diffusion tensor imaging. NeuroImage 47, 618-627 (2009)

9. Peyrat, J.M., Sermesant, M., Pennec, X., Delingette, H., Xu, C., McVeigh, E.R., Ayache, N.: A computational framework for the statistical analysis of cardiac diffusion tensors: Application to a small database of canine hearts. IEEE Trans. on Med. Imaging 26(11), 1500-1514 (2007)

10. Goodlett, C., Davis, B., Jean, R., Gilmore, J., Gerig, G.: Improved correspondence for dti population studies via unbiased atlas building. In: MICCAI, pp. 260-267 (2007)

11. Joshi, S., Davis, B., Jomier, M., Gerig, G.: Unbiased diffeomorphic atlas construction for computational anatomy. NeuroImage 23, S151-S160 (2004)

12. Zhang, H., Yushkevich, P.A., Rueckert, D., Gee, J.C.: Unbiased white matter atlas construction using diffusion tensor images. In: Ayache, N., Ourselin, S., Maeder, A. (eds.) MICCAI 2007, Part II. LNCS, vol. 4792, pp. 211-218. Springer, Heidelberg (2007)

13. Guimond, A., Meunier, J., Thirion, J.: Average brain models: A convergence study. Computer Vision and Image Understanding 77(2), 192-210 (2000)

14. Barmpoutis, A., Vemuri, B.C.: Groupwise registration and atlas construction of 4 th-order tensor fields using the $\mathrm{r}+$ riemannian metric. In: MICCAI, pp. 640-647 (2007)

15. Geng, X., Christensen, G.E., Gu, H., Ross, T.J., Yang, Y.: Implicit reference-based group-wise image registration and its application to structural and functional mri. NeuroImage 47(4), 1341-1351 (2009) 
16. Kindlmann, G., Estepar, R.S.J., Niethammer, M., Haker, S., Westin, C.F.: Geodesic-loxodromes for diffusion tensor interpolation and difference measurement. In: Ayache, N., Ourselin, S., Maeder, A. (eds.) MICCAI 2007, Part I. LNCS, vol. 4791, pp. 1-9. Springer, Heidelberg (2007)

17. Cachier, P., Ayache, N.: Isotropic energies, filters and splines for vector field regularization. Journal of Mathematical Imaging and Vision 20(3), 251-265 (2004)

18. Peyrat, J.M., Delingette, H., Sermesant, M., Xu, C., Ayache, N.: Registration of 4d cardiac ct sequences under trajectory constraints with multichannel diffeomorphic demons. IEEE Trans. on Med. Imaging (2010)

19. Wang, R., Benner, T., Sorensen, A., Wedeen, V.: Diffusion toolkit: A software package for diffusion imaging data processing and tractography. In: Proceedings of the 15th Annual Meeting of ISMRM (2007)

20. Cox, R.W.: AFNI: Software for analysis and visualization of functional magnetic resonance neuroimages. Computers and Biomedical Research 29, 162-173 (1996)

21. Ziyan, U., Sabuncu1, M.R., O'Donnell, L.J., Westin, C.F.: Nonlinear registration of diffusion $\mathrm{mr}$ images based on fiber bundles, pp. 351-358 (2007)

22. Geng, X., Ross, T.J., Zhan, W., Gu, H., Chao, Y.P., Lin, C.P., Christensen, G.E., Schuff, N., Yang, Y.: Diffusion mri registration using orientation. In: Information Processing in Medical Imaging, pp. 626-637 (2009) 